\title{
Comparison of the characteristics of uncommon epidermal growth factor receptor (EGFR) mutations and EGFR-tyrosine kinase inhibitor treatment in patients with non-small cell lung cancer from different ethnic groups
}

\author{
QIAO ZHANG $^{1 *}$, YANYAN CUI $^{2 *}$, JIAN ZHANG $^{3}$, JIAYIDENG KENJIABIEKE $^{1}$ and PATIGULI AERXIDING ${ }^{1}$ \\ ${ }^{1}$ Department of Thoracic Oncology, The Third Clinical Medical College of Xinjiang Medical \\ University, The Affiliated Tumor Hospital Xinjiang Medical University, Urumqi, Xinjiang 830011; \\ ${ }^{2}$ Department of Geriatric, The First Affiliated Hospital, School of Medicine, Shihezi University, Shihezi, Xinjiang 832000; \\ ${ }^{3}$ Outpatient Department, People's Liberation Army 69260 Troops of Medical Team, Urumqi, Xinjiang 830002, P.R. China
}

Received July 30, 2019; Accepted January 10, 2020

DOI: $10.3892 / \mathrm{etm} .2020 .8612$

\begin{abstract}
Patients with epidermal growth factor receptor (EGFR)-sensitive mutations generally have a significantly higher objective response rate (ORR) and longer progression-free survival (PFS) after EGFR-tyrosine kinase inhibitor (TKI) treatment. However, the efficacy of EGFR-TKIs in the case of uncommon EGFR mutations has remained elusive. In the present study, the characteristics of uncommon EGFR mutations and EGFR-TKI treatments were compared in patients with non-small cell lung cancer (NSCLC) from different ethnic groups. A total of 2,984 patients with pathologically confirmed NSCLC encountered between February 2012 to February 2017 at the Affiliated Tumor Hospital of Xinjiang Medical University (Urumqi, China) were enrolled in the present study. The Amplification Refractory Mutation System was adopted to determine EGFR gene expression, compare the ethnic differences in EGFR mutations between Xinjiang Uygur and Han people, analyze the distribution of uncommon mutation types and evaluate the link between clinicopathological features associated with uncommon mutations and the efficacy of EGFR-TKI treatment. There were significant differences in EGFR mutations in lung adenocarcinoma and lung squamous cell carcinoma between patients
\end{abstract}

Correspondence to: Ms. Patiguli Aerxiding, Department of Thoracic Oncology, The Third Clinical Medical College of Xinjiang Medical University, The Affiliated Tumor Hospital Xinjiang Medical University, 789 Suzhou East Street, Urumqi, Xinjiang 830011, P.R. China

E-mail: patiguli1228@163.com

*Contributed equally

Key words: ethnicity, non-small cell lung cancer, epidermal growth factor receptor, uncommon mutation from the Xinjiang Uygur group and the Han group $(\mathrm{P}<0.001)$. The differences in the uncommon EGFR mutations were significant in patients with lung adenocarcinoma $(\mathrm{P}<0.05)$. The most common site of lymph node metastasis in patients with uncommon mutations was the hilar lymph node, supraclavicular/subclavian lymph node, cervical lymph node and mediastinal lymph node; the most common distant metastatic organs were the lung, bone, brain, liver and adrenal gland. Of the uncommon mutations, the most common single mutations were L861Q, G719X and 20ins mutations; the most common double mutation was the S768I and 20ins mutation. The incidence rate of EGFR gene mutations was significantly higher in Han people from Xinjiang than in Uygur people. There were marked differences between individuals regarding the efficacy of EGFR-TKI treatment and the survival time of patients with uncommon EGFR mutations, second-line EGFR-TKIs had a lower ORR and DCR while had a longer mPFS. All of these could provide a basis for the exploration of different regimens for patients with different types of uncommon mutations.

\section{Introduction}

The epidermal growth factor receptor (EGFR) is expressed on the surface of normal epithelial cells and is overexpressed in certain tumor cells. Overexpression of EGFR is linked to the migration and invasion of tumor cells and patient prognosis (1). Distant metastasis is a complex process in which cancer cells leave the primary tumor, invade the lymph and blood system and growing in distant organ sites; each of these steps is closely associated with the biological characteristics of the tumor (2). Lymph node metastasis is the most common metastatic pathway of lung cancer, which affects its stage and prognosis (3). Previous studies have indicated that EGFR mutations are highly associated with ethnicity, gender, adenocarcinoma and smoking status (4-9). With the application of sequencing and PCR technology, EGFR mutations have become the major predictors of the effectiveness of EGFR-tyrosine kinase inhibitor (TKI) targeted therapy (10). Patients on EGFR-TKI 
treatment had an objective response rate (ORR) of $70-80 \%$, median progression-free survival (mPFS) of 9-12 months and overall survival (OS) of 20-32 months, making EGFR-TKI become the first-line treatment for patients with EGFR-mutant non-small cell lung cancer (NSCLC) (9).

EGFR mutations mainly exist in exons 18-21, among which exon 19 deletion mutation, exon $21 \mathrm{~L} 858 \mathrm{R}$ point mutation and exon $20 \mathrm{~T} 790 \mathrm{M}$ mutation are the most common types, which account for $50-90 \%$ of the overall EGFR mutations and are referred to as classical mutations $(8,11)$; other sensitive mutations, referred to as uncommon mutations in clinical practice, include G719X in exon18, L861Q and G719C in exon $21(12,13)$. Uncommon mutations and common mutations have been indicated to have similar clinicopathological features, but uncommon mutations have a lower sensitivity and a lesser response to EGFR-TKI treatment $(14,15)$. Previous studies have suggested that for EGFR-TKI treatment, NSCLC patients with uncommon mutations are less responsive than those with common mutations, as indicated by their shorter PFS and lower ORR after EGFR-TKI treatment $(16,17)$. However, due to the low incidence of uncommon mutations and the partial overlap of the incidence of the two types of mutations, the reasons for the differences in the therapeutic effect of EGFR-TKIs across different ethnicities and mutation types have remained elusive; furthermore, only a small number of relevant studies has been published to date $(18,19)$.

The frequency of EGFR mutation in NSCLC has been documented to differ across ethnic groups and the occurrence was observed to be markedly higher in East-Asian trials compared with that in European studies $(20,21)$. China is a large country by area; Uygur people live between East Asia and the European continent $(20,22)$, but little is known regarding the difference in EGFR mutation rates between Xinjiang Uygur and Han people.

The present study was based on populations of different genetic backgrounds and living habits, i.e., Xinjiang Uygur and Han people. The ADx Amplification Refractory Mutation System (ADx-ARMS) was used to determine EGFR gene expression, compare the ethnic differences in EGFR mutations between Xinjiang Uygur and Han people, analyze the distribution of uncommon mutation types and evaluate the association between the clinicopathological features of uncommon mutations and the efficacy of EGFR-TKI treatment, which aimed to justify the clinical treatment of patients with uncommon EGFR mutations.

\section{Materials and methods}

Patients. A total of 2,984 patients who were hospitalized at the Affiliated Tumor Hospital of Xinjiang Medical University (Urumqi, China) between February 2012 and February 2017 and had been pathologically confirmed as having NSCLC were enrolled in the present study, among whom 29 patients with uncommon mutations were screened out after the ADx-ARMS test for EGFR gene mutations. The study was approved by the Affiliated Tumor Hospital Xinjiang Medical University (Urumqi, China). All patients provided written informed consent prior to enrollment. The inclusion criteria were as follows: i) Pathologically confirmed lung adenocarcinoma; ii) advanced clinical stage (stage IIIB or IV) according to the seventh edition American Joint Committee on Cancer (AJCC) staging system (23); iii) age >18 years; iv) all tissue samples had been tested for EGFR gene expression prior to treatment; v) patients treated with EGFR-TKIs were defined as patients receiving standard treatment with gefitinib, erlotinib and icotinib for at least 30 days; vi) at least one measurable lesion as defined by the Response Evaluation Criteria in Solid Tumors (RECIST) 1.1 (24); vii) clinical stage was determined using the AJCC TNM staging system (VII) (23); viii) regular follow-ups had been performed with complete pathological data. The exclusion criteria were as follows: i) Patients with known drug-resistant EGFR mutations; ii) patients who had received no EGFR-TKI treatment or had not been treated with EGFR-TKI as required; iii) patients who stopped the medication or reduced the dose due to adverse reactions; iv) patients who died from diseases not associated with the disease studied (e.g. heart disease or severe pulmonary infection); v) patients with their follow-up results lost.

Methods. A retrospective analysis was performed on the clinical data of the patients with uncommon mutations $(n=29)$, including gender, age, smoking status, ethnicity, pathological type, TNM stage and primary lesion location. In order to explore whether uncommon EGFR mutations were associated with specific metastases, patients with uncommon mutations were examined for organ and lymph node metastasis. MRI was used to determine brain metastasis and CT to determine other metastases. In addition, the EGFR mutation type, optimal efficacy of targeted therapy, time of disease progression, time of last follow-up and time of death were recorded.

$A D x$-ARMS. The ARMS was adopted to determine EGFR gene expression in the tissue samples of all patients $(n=2,984)$, and all of the tissue samples were obtained during surgery. The ARMS is a highly sensitive real-time PCR-based test system that covers the 29 EGFR mutation hotspots from exon 18 to exon 21 (25). The assay was performed with the MX3000P (Stratagene) real-time PCR system according to the manufacturer's protocol for the ADx EGFR29 Mutation Kit (Amoy Diagnostics). The 25- $\mu 1$ RT-PCR system consisted of $0.4 \mu \mathrm{l}$ of template DNA, $3.6 \mu \mathrm{l}$ of deionized water and $16 \mu \mathrm{l}$ of other reaction components (26). PCR was performed by initial denaturation at $95^{\circ} \mathrm{C}$ for $10 \mathrm{~min}$, followed by 40 cycles of amplification $\left(95^{\circ} \mathrm{C}\right.$ for $30 \mathrm{sec}$ and $61^{\circ} \mathrm{C}$ for $\left.1 \mathrm{~min}\right)$. The results were analyzed according to the criteria defined by the manufacturer's protocol.

Efficacy evaluation indexes. According to the RECIST, the efficacy was divided into complete response (CR), partial response (PR), stable disease (SD) and progressive disease. From the numbers of patients with $\mathrm{CR}, \mathrm{PR}$ and $\mathrm{SD}$, the following parameters were calculated: $\mathrm{ORR}=\left(\mathrm{n}_{\mathrm{CR}}+\mathrm{n}_{\mathrm{PR}}\right) /$ total number of cases $\times 100 \%$. Disease control rate $(\mathrm{DCR})=\left(\mathrm{n}_{\mathrm{CR}}+\mathrm{n}_{\mathrm{PR}}+\mathrm{n}_{\mathrm{SD}}\right) /$ total number of cases $\mathrm{x} 100 \%$.

Statistical analysis. SPSS (version 21.0; IBM Corp.) was used for data analysis. The $\chi^{2}$ test was used to analyze the differences in the uncommon EGFR mutations between Uygur and Han people. The normally distributed data were expressed as the mean \pm standard deviation, while the non-normally distributed 
Table I. Differences in EGFR gene mutations between Xinjiang Uygur and Han ethnicities.

\begin{tabular}{|c|c|c|c|c|c|c|c|c|}
\hline \multirow[b]{2}{*}{ Ethnicity } & \multicolumn{2}{|c|}{$\begin{array}{c}\text { EGFR } \\
\text { mutation }\end{array}$} & \multirow[b]{2}{*}{ Mutation rate } & \multirow[b]{2}{*}{ P-value } & \multicolumn{2}{|c|}{$\begin{array}{l}\text { Uncommon } \\
\text { EGFR } \\
\text { mutation }\end{array}$} & \multirow[b]{2}{*}{ Mutation rate } & \multirow[b]{2}{*}{ P-value } \\
\hline & + & - & & & + & - & & \\
\hline Adenocarcinoma & & & & $<0.001$ & & & & $<0.05$ \\
\hline Uygur & 42 & 347 & 10.79 & & 1 & 388 & 0.25 & \\
\hline Han & 923 & 355 & 72.22 & & 25 & 1253 & 1.96 & \\
\hline Squamous cell carcinoma & & & & $<0.001$ & & & & 1.0 \\
\hline Uygur & 5 & 148 & 3.26 & & 0 & 153 & 0 & \\
\hline Han & 118 & 1046 & 10.13 & & 3 & 1161 & 0.26 & \\
\hline
\end{tabular}

The percentage value is from the number of patients with a positive common and uncommon EGFR mutation. EGFR common mutation includes: Exon 19 deletion mutation, exon 21 L858R point mutation and exon 20 T790M mutation. Uncommon EGFR mutation includes: Exon 18 G719X mutation, exon 20 20ins mutation, exon 20 S768I mutation and exon 21 L861Q mutation. EGFR, epidermal growth factor receptor.

data were expressed as the median with interquartile range. $\mathrm{P}<0.05$ was considered to indicate statistical significance.

\section{Results}

Differences in EGFR gene mutations between Xinjiang Uygur and Han people. A total of 2,984 patients (542 Uygur people and 2,442 Han people) with stage IIIb/IV NSCLC were enrolled in the study. Among the 542 Uygur people, 298 were male and 244 were female, with a median age of 63 (range, 36 to 89 years). Among the 2,442 Han people, 1018 were male and 1424 were female, with a median age of 66 (ranging from 23 to 87 years). The clinical and pathological features of Xinjiang Uygur and Han ethnicities were showed in Table SI. There were significant differences in EGFR mutations between Xinjiang Uygur and Han people $(\mathrm{P}<0.001)$ : Uygur and Han people with adenocarcinoma had an EGFR mutation rate of 10.79 and $72.22 \%$, respectively, and those with squamous cell carcinoma had an EGFR mutation rate of 3.26 and $10.13 \%$, respectively. The differences in uncommon EGFR mutations were significant between Uygur and Han people with lung adenocarcinoma $(\mathrm{P}<0.05)$, but not significant between those with lung squamous cell carcinoma. Uygur and Han people with lung adenocarcinoma had an uncommon EGFR mutation rate of 0.25 and $1.96 \%$, respectively, and those with lung squamous cell carcinoma had an uncommon EGFR mutation rate of 0 and $0.26 \%$, respectively. The differences in the EGFR gene mutations between Xinjiang Uygur and Han people are presented in Table I.

Clinicopathological features of patients with uncommon mutations. The clinicopathological features of the 29 patients with uncommon mutations (25 were Han people, 4 was Uygur) are presented in Table II. The patients with uncommon mutations consisted of 19 men and 10 women, and 17 were nonsmokers. A total of $3(10.3 \%)$ patients had Squamous cell carcinoma and $26(89.7 \%)$ had Adenocarcinoma. A total of 8 $(27.6 \%)$ patients were classified as at stage IIIB, and $21(72.4 \%)$ at stage IV. A total of 13 patients had the primary tumor in left lung. The most common site of lymph node metastasis in patients with uncommon mutations was the hilar lymph node, supraclavicular/subclavian lymph node, cervical lymph node and mediastinal lymph node; the most common distant metastatic organs were the lung, bone, brain, liver and adrenal gland.

Pathological types of uncommon mutations. Among the 29 patients with uncommon mutations, 16 had single mutations, 11 had double mutations and 2 had triple mutations (Table III).

EGFR-TKI treatments for uncommon mutations. A total of 29 patients with uncommon mutations were treated with EGFR-TKIs, among whom 16 were treated with gefitinib, 12 with erlotinib and 1 with icotinib. The number of patients treated with first-line, second-line and third-line EGFR-TKIs was 16,7 and 6 , respectively. Their clinicopathological features and outcomes are presented in Tables IV-VI.

The ORRs, DCRs and mPFS on treatment with first-line, second-line and third-line EGFR-TKIs are provided in Table VII, second-line EGFR-TKIs have a lower ORR and DCR while had a longer mPFS.

The mPFS of all patients with uncommon mutations who received EGFR-TKIs ranged from 5.5 to 2.7 months (first-line, second-line and third-line EGFR-TKIs was 5.5, 4.0 and 2.7 months, respectively), with no significant differences observed (Fig. 1), but the result indicated that the mPFS was shortened with the increasing lines of EGFR-TKIs.

\section{Discussion}

Lung cancer is one of the deadliest malignant tumor types in the world. EGFR is a cell proliferation and signaling receptor for epidermal growth factors. Furthermore, it is a receptor tyrosine kinase that is frequently overexpressed and has a central role in the development of $\operatorname{NSCLC}(27,28)$. Studies on treatments targeting EGFR have opened up novel avenues for the treatment of lung malignancies, but sensitivity to treatment 
Table II. Clinicopathological features of patients with uncommon mutations $(n=29)$.

\begin{tabular}{|c|c|}
\hline Characteristics & Value $(\%)$ \\
\hline \multicolumn{2}{|l|}{ Sex } \\
\hline Male & $19(65.5)$ \\
\hline Female & $10(34.5)$ \\
\hline Median age (years) & $68(38-82)$ \\
\hline \multicolumn{2}{|l|}{ Smoking } \\
\hline Smoker & $12(41.3)$ \\
\hline Non-smoker & $17(58.7)$ \\
\hline $\begin{array}{l}\text { Average smoking index } \\
\text { (patients with smoking habits) }\end{array}$ & $495.8 \pm 23.7$ \\
\hline Smoking index interval & $100-900$ \\
\hline \multicolumn{2}{|l|}{ Ethnicity } \\
\hline Han & $25(86.2)$ \\
\hline Uygur & $4(13.8)$ \\
\hline \multicolumn{2}{|l|}{ Pathological type } \\
\hline Squamous cell carcinoma & $3(10.3)$ \\
\hline Adenocarcinoma & $26(89.7)$ \\
\hline \multicolumn{2}{|l|}{ Stage } \\
\hline IIIB & $8(27.6)$ \\
\hline IV & $21(72.4)$ \\
\hline \multicolumn{2}{|l|}{ Primary tumor location } \\
\hline Left lung & $13(44.8)$ \\
\hline Right lung & $16(55.2)$ \\
\hline \multicolumn{2}{|l|}{ Metastatic organ } \\
\hline Lung & $14(48.3)$ \\
\hline Bone & $10(34.5)$ \\
\hline Brain & $9(31.0)$ \\
\hline Liver & $5(17.2)$ \\
\hline Adrenal gland & $4(13.8)$ \\
\hline Other & $5(17.2)$ \\
\hline \multicolumn{2}{|l|}{ Lymph node metastasis } \\
\hline Hilar lymph node & $8(27.6)$ \\
\hline Clavicular lymph node & $5(17.2)$ \\
\hline Cervical lymph node & $3(10.3)$ \\
\hline Mediastinal lymph node & $1(3.4)$ \\
\hline Other & $2(6.9)$ \\
\hline
\end{tabular}

Values are expressed as $\mathrm{n}(\%)$ or the median (range).

is significantly associated with EGFR mutation types. Exon 19 deletion and exon 21 L858R mutation are the most common types, which account for almost $90 \%$ of all EGFR mutations in lung cancer $(29,30)$. Due to the small proportion of patients with uncommon mutations, evidence-based medical evidence is only available from retrospective studies and case reports with small samples. Certain studies have indicated that the proportion of patients with uncommon mutations receiving first-line EGFR-TKIs is up to $85.7 \%$, and that proportion is higher for patients with uncommon mutations combined with 19-DEL and L858R complex mutations. Furthermore, the ORR,
Table III. Distribution of uncommon EGFR mutations by type.

\begin{tabular}{lcl}
\hline EGFR mutation & Mutant exon & No. of patients $(\%)$ \\
\hline G719X & 18 & $5(17.2)$ \\
20ins & 20 & $4(13.8)$ \\
S768I & 20 & $2(6.9)$ \\
L861Q & 21 & $5(17.2)$ \\
G719X+20ins & $18+20$ & $1(3.4)$ \\
G719X+T790M & $18+20$ & $2(6.9)$ \\
G719X+L861Q & $18+21$ & $2(17.2)$ \\
19Del+S768I & $19+20$ & $1(3.4)$ \\
S768I+20ins & 20 & $4(13.8)$ \\
S768I+L858R & $20+21$ & $1(3.4)$ \\
G719X+S768I+20ins & $18+20$ & $1(3.4)$ \\
G719X+L861Q+L858R & $18+21$ & $1(3.4)$ \\
\hline
\end{tabular}

EGFR, epidermal growth factor receptor; No., number; ins, insertion; del, deletion.

mPFS and OS of patients with certain compound mutations are similar to those of patients with common mutations (31).

In the present study, 2,984 patients of Uygur and Han ethnicities with stage IIIB/IV NSCLC in Xinjiang were retrospectively analyzed and the results indicated that Uygur and Han people exhibited significant differences in EGFR mutations in adenocarcinoma and squamous cell carcinoma. A meta-analysis by Wang and Wang (26) suggested that the overall EGFR mutation rate of Chinese patients was $37.5 \%$ and $\mathrm{Wu}$ et al $(32,33)$ reported that $37.9 \%$ of Chinese NSCLS patients had EGFR mutations. However, the EGFR mutation rate of Han people in the present study was $72.22 \%$, which was higher than that in other studies. The reason for this may be the use of ADx-ARMS, a different and more sensitive method, in the present study. The differences in the uncommon EGFR mutations were significant between Uygur and Han people with lung adenocarcinoma, but not significant between the two ethnic groups with lung squamous cell carcinoma. A total of 2,984 patients with EGFR mutations were enrolled, among whom 29 harbored uncommon mutations. It was indicated that the proportion of patients harboring uncommon EGFR mutations was not significantly different across different genders and smoking statuses, which was similar to the results obtained by Sonobe et al (34). The most common lymph node metastasis sites in patients with uncommon mutations were hilar lymph node, supraclavicular/subclavian lymph node, cervical lymph node and mediastinal lymph node, and the most common distant metastatic organs were the lung, bone, brain, liver and adrenal gland. Comparison of the efficacy of EGFR-TKIs in patients with uncommon EGFR mutations revealed that patients on treatment with first-line EGFR-TKIs had an ORR of $43.75 \%$, a DCR of $50 \%$ and mPFS of 5.5 months; the ORR and PFS of patients on treatment with first-line EGFR-TKIs were inferior to those in patients with classical mutations, and were also inferior to the previous research of certain patients with uncommon mutations, but were superior to those with wild-type EGFRs. The second-line EGFR-TKIs had an ORR of $28.57 \%$, a DCR of 
Table IV. Clinicopathological features and outcomes of 16 patients on first-line EGFR-TKI treatment.

\begin{tabular}{rccccclr}
\hline Pt. ID & Sex & Age (years) & Smoking & Stage & Histology & EGFR mutation & TKI response \\
\hline 1 & F & 53 & Y & IV & ADC & G719X & PR \\
2 & F & 82 & Y & IV & ADC & G719X+20ins & PD \\
3 & M & 71 & N & IV & ADC & 20ins & PD \\
4 & M & 67 & N & IV & ADC & S768I & PD \\
5 & M & 65 & N & IIIB & ADC & G719X & PR \\
6 & F & 54 & N & IV & ADC & L861Q & 6 \\
7 & F & 61 & Y & IV & ADC & G719X+T790M & PD \\
8 & M & 73 & Y & IV & ADC & G719X+L861Q & PR \\
9 & M & 57 & N & IV & ADC & L861Q & 1 \\
10 & M & 63 & N & IIIB & ADC & 19Del+T790M & SD \\
11 & M & 82 & Y & IIIB & ADC & G719X & 9 \\
12 & M & 68 & N & IV & ADC & L861Q & PR \\
13 & F & 71 & Y & IV & ADC & S768I+L858R & PR \\
14 & M & 66 & N & IV & ADC & 19Del+S768I & PD \\
15 & M & 52 & N & IIIB & ADC & 20ins & PR \\
16 & F & 48 & Y & IV & ADC & S768I+20ins & 17 \\
\hline
\end{tabular}

EGFR-TKI, epidermal growth factor receptor tyrosine kinase inhibitor; Pt. ID, patient identification number; PFS, progression-free survival; M, male; F, female; Y, yes; N, no; ADC, adenocarcinoma; ins, insertion; del, deletion; PR, partial response; PD, progressive disease; SD, stable disease.

Table V. Clinicopathological features and outcomes of 7 patients on second-line EGFR-TKI treatment.

\begin{tabular}{lccccclcc}
\hline Pt. ID & Sex & Age (years) & Smoking & Stage & Histology & \multicolumn{1}{c}{ EGFR mutation } & TKI response & PFS \\
\hline 1 & M & 38 & N & IIIB & ADC & L861Q & SD & 6 \\
2 & M & 64 & N & IV & ADC & G719X & PD & 5 \\
3 & M & 71 & N & IV & ADC & G719X+S768I+20ins & PD & 2 \\
4 & M & 68 & Y & IIIB & ADC & G719X+L861Q & PR & 5 \\
5 & M & 55 & N & IV & SCC & S768I & PD & 2 \\
6 & F & 81 & Y & IIIB & ADC & S768I+20ins & PD & 2 \\
7 & F & 68 & N & IV & ADC & G719X+L861Q+L858R & PR & 6 \\
\hline
\end{tabular}

EGFR-TKI, epidermal growth factor receptor tyrosine kinase inhibitor; Pt. ID, patient identification number; PFS, progression-free survival; M, male; F, female; Y, yes; N, no; ADC, adenocarcinoma; SCC, squamous cell carcinoma; ins, insertion; SD, stable disease; PD, progressive disease; PR, partial response.

Table VI. Clinicopathological features and outcomes of 6 patients on third-line EGFR-TKI treatment.

\begin{tabular}{lcccccccc}
\hline Pt. ID & Sex & Age (years) & Smoking & Stage & Histology & EGFR mutation & TKI response & PFS \\
\hline 1 & F & 53 & N & IV & SCC & L861Q & PD & 2 \\
2 & F & 43 & Y & IIIB & ADC & G719X & PR & 4 \\
3 & M & 71 & N & IV & ADC & 20ins & PR & 5 \\
4 & M & 67 & Y & IV & SCC & S768I+20ins & PD & 1 \\
5 & M & 65 & N & IV & ADC & 20ins & SD & 2 \\
6 & M & 54 & Y & IV & ADC & S768I+20ins & PD & 2 \\
\hline
\end{tabular}

EGFR-TKI, epidermal growth factor receptor tyrosine kinase inhibitor; Pt. ID, patient identification number; PFS, progression-free survival; M, male; F, female; Y, yes; N, no; ADC, adenocarcinoma; SCC, squamous cell carcinoma; ins, insertion; PD, progressive disease; PR, partial response; $\mathrm{SD}$, stable disease. 
Table VII. Summary of short-term outcomes of 29 patients with uncommon mutations on EGFR-TKI treatment.

\begin{tabular}{|c|c|c|c|c|c|c|c|c|}
\hline EGFR-TKI treatment & Cases (n) & $\mathrm{CR}(\%)$ & PR $(\%)$ & $\mathrm{SD}(\%)$ & $\mathrm{PD}(\%)$ & ORR (\%) & $\operatorname{DCR}(\%)$ & mPFS (months) \\
\hline First-line & 16 & $0(0.0)$ & $7(43.8)$ & $1(6.3)$ & $8(50.0)$ & 43.8 & 50.0 & 5.5 \\
\hline Second-line & 7 & $0(0.0)$ & $2(28.6)$ & $1(14.3)$ & $4(57.1)$ & 28.6 & 42.9 & 4.0 \\
\hline Third-line & 6 & $0(0.0)$ & $2(33.3)$ & $1(16.7)$ & $3(50.0)$ & 33.3 & 50.0 & 2.7 \\
\hline
\end{tabular}

mPFS, median progression-free survival; EGFR-TKI, epidermal growth factor receptor tyrosine kinase inhibitor; PR, partial response; $\mathrm{PD}$, progressive disease; $\mathrm{SD}$, stable disease; $\mathrm{CR}$, complete response; ORR, objective response rate; DCR, disease control rate.

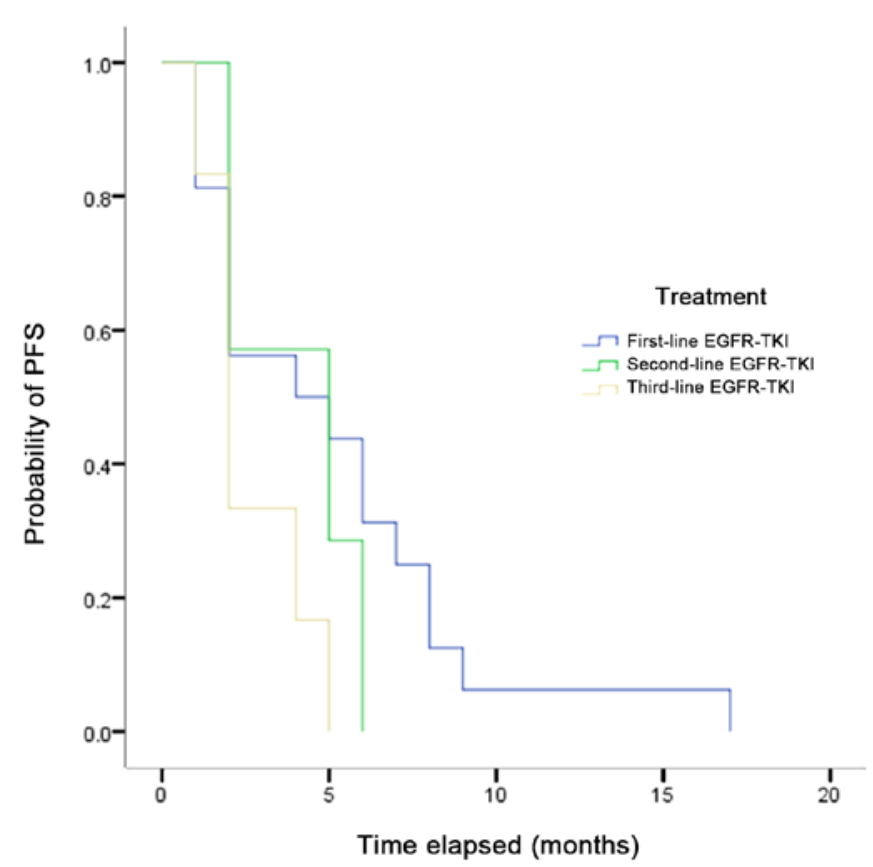

Figure 1. PFS analysis of patients with uncommon EGFR mutations treated with EGFR-TKIs. PFS, progression-free survival; EGFR-TKI, epidermal growth factor receptor tyrosine kinase inhibitor.

$42.85 \%$ and mPFS of 4.0 months. The three-line EGFR-TKIs had an ORR of $33.33 \%$, a DCR of $50.00 \%$ and mPFS of 2.7 months. From this observation, it may be concluded that the mPFS was shortened with the increasing lines of EGFR-TKIs, which may be linked to the changes in patients' physical state, drug tolerance and EGFR mutation kurtosis.

In the present study, the most common mutation site was G719X. Among the 29 patients, 5 harbored G719X single mutations and 6 harbored compound mutations. There was a point mutation of G719 at exon 18: The glycine at position 719 was replaced by serine, alanine or cysteine (G719S/A/C). Previous studies have suggested that the affinity for ATP of the G719 mutant is between that of wild-type EGFR and L858R (35). According to one study, patients with G791X single mutations had an ORR of $36.8 \%$ (36), but it has also been reported that patients with G719 mutations, whether single or double, had an ORR of $53.3 \%$ and mPFS of 8.1 months (37). In the present study, 5 patients with G719X mutations had an ORR of $80 \%$ and mPFS of 6 months, and 6 patients with compound mutations had an ORR of $50 \%$ and mPFS of 3.5 months. The best response achieved was PR; the ORR and PFS for patients with single mutations were superior to those of patients with compound mutations but inferior to those of patients with classical mutations. It may be suggested that certain patients with compound mutations harbored drug-resistant mutations whose affinity for ATP was lower than that of G719 single mutations and the possibility of bypass interference cannot be excluded.

L861Q, as a mutation at position 861 on exon 20 of the EGFR, was another common mutation site in the present study. Among the 29 patients, 5 had L861Q single mutations and 3 had compound mutations. A previous study reported that L861Q accounted for 2\% of EGFR mutations (38); Yoshida et al (39) indicated that patients with L861Q mutation were resistant to first-line EGFR-TKIs, but certain other studies suggested that certain EGFR-TKIs were effective for the treatment of L816Q mutation with the efficacy being inferior to that for L858R and G719 mutations $(13,25)$. In the NEJ002 study, the efficacy of gefitinib was retrospectively analyzed in 7 patients with uncommon G719X mutations and 3 patients with uncommon L8861Q. It was indicated that the median OS of the patients with uncommon sensitive mutations in the gefitinib group was significantly shorter than that in the classical sensitive mutation group, while the median OS was not significantly different between patients with uncommon sensitive mutations and those with common sensitive mutations in the chemotherapy group (40). In the present study, the ORR was $40 \%$ and the mPFS was 6.2 months in 5 patients with L861Q treated with first-line EGFR-TKIs.

However, it should be noted that cohort size $(n=2,984)$ of the present study was relatively small, which may have affected the uncommon EGRF mutation rate between Uygur and Han people. The number of patients with uncommon EGFR mutations among the Uygur people was too small, so large samples should be carried out in the future to further verify the results of the present study.

In conclusion, the incidence of EGFR gene mutations is significantly higher in Han people who live in Xinjiang than in Uygur people. The uncommon EGFR mutations may be divided into different subtypes, which may result in different outcomes and survival time of patients on treatment with EGFR-TKIs. The mechanism of action may include the involvement of different signaling pathways. In general, patients with uncommon EGFR mutations have a lower ORR and shorter PFS than those with classical mutations, but certain patients with uncommon EGFR mutations have a higher ORR and longer PFS than patients with wild-type mutations. Therefore, patients with different types of uncommon mutations should be treated with different regimens. The specific mechanism of action requires further investigation. 


\section{Acknowledgements}

Not applicable.

\section{Funding}

This study was supported by the Xinjiang Uygur Autonomous Region Municipal Natural Science Foundation (grant no. 2016D01C376) and the Youth Medical Science and Technology Talents Special Scientific Research Project of the Health and Family Planning Commission in Xinjiang Uygur Autonomous Region (grant no. WJWY-201907).

\section{Availability of data and materials}

The datasets used and/or analyzed during the present study are available from the corresponding author on reasonable request.

\section{Authors' contributions}

QZ contributed to the conception and design of the study. QZ, YC and JZ were responsible for the collection and analysis of the data and wrote the manuscript. JK and PA were involved in the data analysis and interpretation. All authors read and approved the final manuscript.

\section{Ethics approval and consent to participate}

The study was approved by the Ethics Committee of The Affiliated Tumor Hospital Xinjiang Medical University (G-201832). The patients provided written informed consent for the participation of the present study and publication of any associated data.

\section{Patient consent for publication}

Not applicable.

\section{Competing interests}

The authors declare that they have no competing interests.

\section{References}

1. Paez JG, Jänne PA, Lee JC, Tracy S, Greulich H, Gabriel S, Herman P, Kaye FJ, Lindeman N, Boggon TJ, et al: EGFR mutations in lung cancer: Correlation with clinical response to gefitinib therapy. Science 304: 1497-500, 2004.

2. Brooks DL, Wakefield L and Steeg P: Abstract 4859: The role of LPAR 1 and fibrosis in breast cancer metastasis. Cancer Res 77: 4859-4859, 2017.

3. Wang Y, Zhu B, Ye M and Chen Z: Study on relationship between VEGF-C and lymphangiogenesis and lymph node metastasis in non-small cell lung cancer. Zhongguo Fei Ai Za Zhi 9: 182-186, 2006 (In Chinese).

4. Mok TS, Wu YL, Thongprasert S, Yang CH, Chu DT, Saijo N, Sunpaweravong P, Han B, Margono B, Ichinose Y, et al: Gefitinib or carboplatin-paclitaxel in pulmonary adenocarcinoma. N Engl J Med 361: 947-957, 2009.

5. Mitsudomi T, Morita S, Yatabe Y, Negoro S, Okamoto I, Tsurutani J, Seto T, Satouchi M, Tada H, Hirashima T, et al: Gefitinib versus cisplatin plus docetaxel in patients with non-small-cell lung cancer harbouring mutations of the epidermal growth factor receptor (WJTOG3405): An open label, randomised phase 3 trial. Lancet Oncol 11: 121-128, 2010.
6. Zhou C, Wu YL, Chen G, Feng J, Liu XQ, Wang C, Zhang S, Wang J, Zhou S, Ren S, et al: Erlotinib versus chemotherapy as first-line treatment for patients with advanced EGFR mutation-positive non-small-cell lung cancer (OPTIMAL, CTONG-0802): A multicentre, open-label, randomised, phase 3 study. Lancet Oncol 12: 735-742, 2011.

7. Arrieta O, Campos-Parra AD, Zuloaga C, Avilés A, SánchezReyes R, Manríquez MEV, Covián-Molina E, Martínez-Barrera L, Meneses A, Cardona A and Borbolla-Escoboza JR: Clinical and pathological characteristics, outcome and mutational profiles regarding non-small-cell lung cancer related to wood-smoke exposure. J Thorac Oncol 7: 1228-1234, 2012.

8. Arrieta O, Cardona AF, Federico Bramuglia G, Gallo A Campos-Parra AD, Serrano S, Castro M, Avilés A, Amorin E, Kirchuk R, et al: Genotyping non-small cell lung cancer (NSCLC) in Latin America. J Thorac Oncol 6: 1955-1959, 2011.

9. Lee HJ, Kim YT, Kang CH, Zhao B, Tan Y, Schwartz LH, Persigehl T, Jeon YK and Chung DH: Epidermal growth factor receptor mutation in lung adenocarcinomas: Relationship with CT characteristics and histologic subtypes. Radiology 268: 254-264, 2013.

10. Yamane H, Ochi N, Yasugi M, Tabayashi T, Yamagishi T, Monobe Y, Hisamoto A, Kiura K and Takigawa N: Docetaxel for non-small-cell lung cancer harboring the activated EGFR mutation with T790M at initial presentation. Onco Targets Ther 6: 155-160, 2013.

11. Rosell R, Moran T, Queralt C, Porta R, Cardenal F, Camps C, Majem M, Lopez-Vivanco G, Isla D, Provencio M, et al: Screening for epidermal growth factor receptor mutations in lung cancer. N Engl J Med 361: 958-967, 2009.

12. Shigematsu H, Lin L, Takahashi T, Nomura M, Suzuki M, Wistuba II, Fong KM, Lee H, Toyooka S, Shimizu N, et al: Clinical and biological features associated with epidermal growth factor receptor gene mutations in lung cancers. J Natl Cancer Inst 97: 339-346, 2005.

13. Pallis AG, Voutsina A, Kalikaki A, Souglakos J, Briasoulis E, Murray S, Koutsopoulos A, Tripaki M, Stathopoulos E, Mavroudis D and Georgoulias V: 'Classical' but not 'other' mutations of EGFR kinase domain are associated with clinical outcome in gefitinib-treated patients with non-small cell lung cancer. Br J Cancer 97: 1560-1566, 2007.

14. Frega S, Lorenzi M, Fassan M, Indraccolo S, Calabrese F, Favaretto A, Bonanno L, Polo V, Zago G, Lunardi F, et al: Clinical features and treatment outcome of non-small cell lung cancer (NSCLC) patients with uncommon or complex epidermal growth factor receptor (EGFR) mutations. Oncotarget 8: 32626-32638, 2017.

15. Xu J, Jin B, Chu T, Dong X, Yang H, Zhang Y, Wu D, Lou Y, Zhang $X$, Wang $H$ and Han B: EGFR tyrosine kinase inhibitor (TKI) in patients with advanced non-small cell lung cancer (NSCLC) harboring uncommon EGFR mutations: A real-world study in China. Lung Cancer 96: 87-92, 2016.

16. Chen D, Song Z and Cheng G: Clinical efficacy of first-generation EGFR-TKIs in patients with advanced non-small-cell lung cancer harboring EGFR exon 20 mutations. Onco Targets Ther 9: 4181-4186, 2016.

17. Kuiper JL, Hashemi SMS, Thunnissen E, Snijders PJF, Grünberg K, Bloemena E, Sie D, Postmus PE, Heideman DA and Smit EF: Non-classic EGFR mutations in a cohort of Dutch EGFR-mutated NSCLC patients and outcomes following EGFR-TKI treatment. Br J Cancer 115: 1504-1512, 2016.

18. Zhou $\mathrm{J}$ and Ben S: Comparison of therapeutic effects of EGFR-tyrosine kinase inhibitors on 19Del and L858R mutations in advanced lung adenocarcinoma and effect on cellular immune function. Thoracic Cancer 9: 228-233, 2018

19. Li XY, Wu JZ, Cao HX, Ma R, Wu JQ, Zhong YJ and Feng JF: Blockade of DNA methylation enhances the therapeutic effect of gefitinib in non-small cell lung cancer cells. Oncol Rep 29: 1975-1982, 2013.

20. Rahman S, Kondo N, Yoneda K, Takuwa T, Hashimoto M, Orui H, Okumura Y, Tanaka F, Kumamoto K, Mostafa MG, et al: Frequency of epidermal growth factor receptor mutations in Bangladeshi patients with adenocarcinoma of the lung. Int J Clin Oncol 19: 45-49, 2014.

21. Smits AJ, Kummer JA, Hinrichs JW, Herder GJ, Scheidel-Jacobse KC, Jiwa NM, Ruijter TE, Nooijen PT, Looijen-Salamon MG, Ligtenberg MJ, et al: EGFR and KRAS mutations in lung carcinomas in the Dutch population: Increased EGFR mutation frequency in malignant pleural effusion of lung adenocarcinoma. Cell Oncol (Dodr) 35: 189-196, 2012. 
22. Zhou D: Detection of EGFR mutation by DHPLC analysis in Chinese NSCLC and colorectal carcinoma patients and its clinical implication. Oncol Prog, 2006.

23. Edge SB and Compton CC: The American Joint Committee on Cancer: The 7th edition of the AJCC Cancer staging manual and the future of TNM. Ann Surg Oncol 17: 1471-1474, 2010.

24. Yoshida S, Miyata Y, Ohtsu A, Boku N, Shirao K and Shimada Y: Significance of and problems in adopting response evaluation criteria in solid tumor RECIST for assessing anticancer effects of advanced gastric cancer. Gastric Cancer 3: 128-133, 2000.

25. Machnicki MM, Glodkowska-Mrowka E, Lewandowski T, Ploski R, Wlodarski P and Stoklosa T: ARMS-PCR for detection of BRAF V600E hotspot mutation in comparison with Real-Time PCR-based techniques. Acta Biochim Pol 60: 57-64, 2013.

26. Chu H, Zhong C, Xue G, Liang X, Wang J, Liu Y, Zhao S, Zhou Q and Bi J: Direct sequencing and amplification refractory mutation system for epidermal growth factor receptor mutations in patients with non-small cell lung cancer. Oncol Rep 30: 2311-2315, 2013

27. Ohsaki Y, Tanno S, Fujita Y, Toyoshima E, Fujiuchi S Nishigaki Y, Ishida S, Nagase A, Miyokawa N, Hirata S and Kikuchi K: Epidermal growth factor receptor expression correlates with poor prognosis in non-small cell lung cancer patients with p53 overexpression. Oncol Rep 7: 603-610, 2000

28. Nicholson RI, Gee JM and Harper ME: EGFR and cancer prognosis. Eur J Cancer 37 (Suppl 4): S9-S15, 2001.

29. Lynch TJ, Bell DW, Sordella R, Gurubhagavatula S, Okimoto RA, Brannigan BW, Harris PL, Haserlat SM, Supko JG, Haluska FG, et al: Activating mutations in the epidermal growth factor receptor underlying responsiveness of non-small-cell lung cancer to gefitinib. N Engl J Med 350: 2129-2139, 2004.

30. Sharma SV, Bell DW, Settleman J and Haber DA: Epidermal growth factor receptor mutations in lung cancer. Nat Rev Cancer 7: 169-181, 2007.

31. Kobayashi S, Canepa HM, Bailey AS, Nakayama S, Yamaguchi N, Goldstein MA, Huberman MS and Costa DB: Compound EGFR mutations and response to EGFR tyrosine kinase inhibitors. J Thorac Oncol 8: 45-51, 2013.

32. Wang $\mathrm{S}$ and Wang Z: EGFR mutations in patients with non-small cell lung cancer from mainland China and their relationships with clinicopathological features: A meta-analysis. Int J Clin Exp Med 7: 1967, 2014.
33. Wu M, Zhao J, Song SW, Zhuo M, Wang X, Bai H, Wang S, Yang L, An T, Zhang Y, et al: EGFR mutations are associated with prognosis but not with the response to front-line chemotherapy in the Chinese patients with advanced non-small cell lung cancer. Lung Cancer 67: 343-347, 2010.

34. Sonobe M, Manabe T, Wada H and Tanaka F: Mutations in the epidermal growth factor receptor gene are linked to smoking-independent, lung adenocarcinoma. Br J Cancer 93: 355-363, 2005.

35. Yun CH, Boggon TJ, Li Y, Woo MS, Greulich H, Meyerson M and Eck MJ: Structures of lung cancer-derived EGFR mutants and inhibitor complexes: Mechanism of activation and insights into differential inhibitor sensitivity. Cancer Cell 11: 217-227, 2007.

36. Chiu CH, Yang CT, Shih JY, Huang MS, Su WC, Lai RS, Wang CC, Hsiao SH, Lin YC, Ho CL, et al: Epidermal growth factor receptor tyrosine kinase inhibitor treatment response in advanced lung adenocarcinomas with G719X/L861Q/S768I mutations. J Thorac Oncol 10: 793-799, 2015.

37. Wu JY, Yu CJ, Chang YC, Yang CH, Shih JY and Yang PC: Effectiveness of tyrosine kinase inhibitors on 'uncommon' epidermal growth factor receptor mutations of unknown clinical significance in non-small cell lung cancer. Clin Cancer Res 17: 3812-3821, 2011.

38. Mitsudomi T and Yatabe Y: Epidermal growth factor receptor in relation to tumor development: EGFR gene and cancer. FEBS J 277: 301-308, 2010 .

39. Yoshida T, Ishii G, Goto K, Neri S, Hashimoto H, Yoh K, Niho S, Umemura S, Matsumoto S, Ohmatsu H, et al: Podoplanin-positive cancer-associated fibroblasts in the tumor microenvironment induce primary resistance to EGFR-TKIs in lung adenocarcinoma with EGFR mutation. Clin Cancer Res 21: 642-651, 2015.

40. Watanabe S, Minegishi Y, Yoshizawa H, Maemondo M, Inoue A, Sugawara S, Isobe H,Harada M, Ishii Y, Gemma A, et al: Effectiveness of gefitinib against non-small-cell lung cancer with the uncommon EGFR mutations G719X and L861Q. J Thorac Oncol 9: 189-194, 2014.

c) (7) $(-)$ This work is licensed under a Creative Commons

cc) ${ }_{\mathrm{EY}}$ NO ND Attribution-NonCommercial-NoDerivatives 4.0 International (CC BY-NC-ND 4.0) License. 